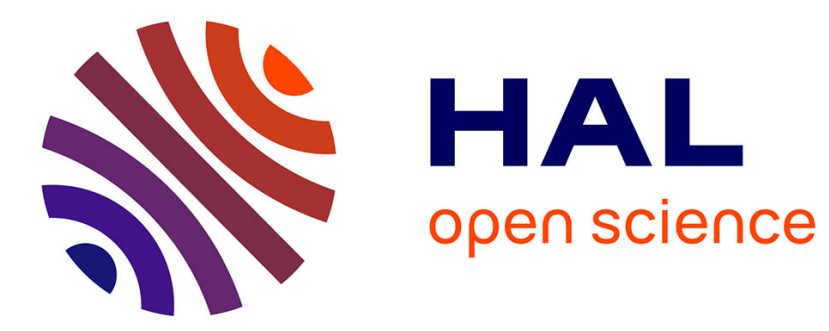

\title{
Targeted delivery of silver nanoparticles and alisertib: in vitro and in vivo synergistic effect against glioblastoma
}

Erica Locatelli, M. Naddaka, Chiara Uboldi, George Loudos, E. Fragogeorgi,

Valerio Molinari, Andrea Pucci, Theodoros Tsotakos, Dimitrios Psimadas, Jessica Ponti, et al.

\section{To cite this version:}

Erica Locatelli, M. Naddaka, Chiara Uboldi, George Loudos, E. Fragogeorgi, et al.. Targeted delivery of silver nanoparticles and alisertib: in vitro and in vivo synergistic effect against glioblastoma. Nanomedicine, 2014, 9 (6), pp.839-849. 10.2217/NNM.14.1 . hal-02353845

\section{HAL Id: hal-02353845 \\ https://hal.science/hal-02353845}

Submitted on 7 Nov 2019

HAL is a multi-disciplinary open access archive for the deposit and dissemination of scientific research documents, whether they are published or not. The documents may come from teaching and research institutions in France or abroad, or from public or private research centers.
L'archive ouverte pluridisciplinaire HAL, est destinée au dépôt et à la diffusion de documents scientifiques de niveau recherche, publiés ou non, émanant des établissements d'enseignement et de recherche français ou étrangers, des laboratoires publics ou privés. 


\title{
Targeted delivery of silver nanoparticles and alisertib: in vitro and in vivo synergistic effect against glioblastoma
}

\begin{abstract}
Aim: Targeted biocompatible nanoplatforms presenting multiple therapeutic functions have great potential for the treatment of cancer. Materials \& methods: Multifunctional nanocomposites formed by polymeric nanoparticles (PNPs) containing two cytotoxic agents - the drug alisertib and silver nanoparticles - were synthesized. These PNPs have been conjugated with a chlorotoxin, an active targeting 36-amino acid-long peptide that specifically binds to MMP-2, a receptor overexpressed by brain cancer cells. Results: The individual and synergistic activity of these two cytotoxic agents against glioblastoma multiforme was tested both in vitro and in vivo. The induced cytotoxicity in a human glioblastoma-astrocytoma epithelial-like cell line (U87MG) was studied in vitro through a trypan blue exclusion test after 48 and $72 \mathrm{~h}$ of exposure. Subsequently, the PNPs' biodistribution in healthy animals and their effect on tumor reduction in tumor-bearing mice were studied using PNPs radiolabeled with ${ }^{99 \mathrm{mT}}$. Conclusion: Tumor reduction was achieved in vivo when using silver/alisertib@PNPs-chlorotoxin.
\end{abstract}

Original submitted 8 April 2013; Revised submitted 19 December 2013

Keywords: alisertib $\bullet$ cancer $\bullet$ glioblastoma $\bullet$ nanoprecipitation $\bullet$ organic coating - polymeric nanoparticle $\bullet$ radiolabeling $\bullet$ silver nanoparticle $\bullet$ toxicity $\bullet$ tumor reduction

Glioblastoma multiforme (GBM) is the most common and deadliest of malignant primary brain tumors in adults and is one of a group of tumors referred to as gliomas. Classified as a grade IV (most serious) astrocytoma, its prognosis is bleak - the median survival time without treatment is 3 months [1]. The number of new diagnoses made annually is two to three per 100,000 people in the USA and Europe. GBM accounts for $12-15 \%$ of all intracranial tumors and $50-60 \%$ of astrocytic tumors [2]. The standard treatment is surgery, followed by radiation therapy or combined radiation therapy and chemotherapy, but surgical removal of such tumors only prolongs the typical patient's survival by less than a year. Some drugs have been used for treatment of adult patients with newly diagnosed GBM. The carmustine implant with polifeprosan 20 [3], temozolomide [4] and bevacizumab [5] have been approved by the US FDA to date. There are several trials that involve many types of therapy, including immunotherapy, antiangiogenic therapy, gene and viral therapy, cancer stem cell therapy, and targeted therapy (personalized medicine) [6,7,101]. Therefore, the quest for new drugs and new delivery systems for targeted therapy is still ongoing and could give new hope to fight GBM.

Nanomedicine is the application of nanotechnology to medicine, and the exploitation of nanoplatforms for cancer treatment holds great promise $[8]$ due to the possibility of tailoring the synthesis of nanoparticles (NPs) in order to produce particles with narrow size distributions and cavities where drugs can be incorporated [9,10]. To date, there is a lot of evidence that these nanocarrier materials are capable of improving the efficiency of therapeutics through well-established targeted
Erica Locatelli', Maria Naddaka', Chiara Uboldi²,

George Loudos ${ }^{3}$,

Eirini Fragogeorgi ${ }^{3}$, Valerio

Molinari', Andrea Pucci',

Theodoros Tsotakos ${ }^{3}$,

Dimitrios Psimadas ${ }^{3}$,

Jessica Ponti ${ }^{2} \&$ Mauro Comes

Franchini ${ }^{*}, 1$

'Department of Industrial Chemistry,

"TosoMontanari", University of Bologna, Via Risorgimento 4, 40136, Bologna, Italy

${ }^{2}$ European Commission, Joint Research

Centre, Institute for Health \& Consumer

Protection, Nanobiosciences Unit, Via E

Fermi 2749, 21027 Ispra, VA, Italy

${ }^{3}$ Department of Biomedical Technology

Engineering, Technological Educational Institute of Athens, Aghiou Spyridonos 28,

12210, Egaleo, Greece

*Author for correspondence:

mauro.comesfranchini@unibo.it 
drug delivery (TDD) techniques [11]. TDD is based on the idea that tumor cells overexpress many receptors and biomarkers that can be used as targets for selective delivery. Therefore, the development of therapeutic carriers that can deliver high drug payloads, while protecting the encapsulated drug from degradation and reducing off-target toxicities, is currently of significant interest [12]. The first generation of drug-loaded NPs with applications in medicine date back to the 1970s, when nanoscaled liposomes were developed to deliver their cargo to diseased cells in a 'Trojan horse' fashion [13]. Since then, a new generation of TDD vehicles (e.g., polymeric NPs [PNPs]) has emerged [14].

PNPs are optimal nanocarriers for TDD due to their small size and ability to entrap efficaciously drug molecules. These tunable characteristics can help to solve the common problems associated with traditional medicine, such as poor drugs solubility in water and short in vivo lifetime. The main feature of these nanosystems is that their surfaces can be functionalized, exploiting terminal reactive groups, with specific proteins, peptides or monoclonal antibodies that are able to selectively bind a site of action or a particular target tissue without interacting with other cells and, thus, minimizing side effects and enhancing drug efficiency. The poly(lacticco-glycolic acid) (PLGA)-block-PEG-carboxylic acid (PLGA-b-PEG) copolymer is an easy-to-synthesize material that is emerging as one of the most promising system for drug loading and in vivo drug delivery applications. PLGA- $b$-PEG is an amphiphilic polymer that self-assembles to generate a targetable system (due to the presence of terminal $\mathrm{COOH}$ functional groups) in which the hydrophobic PLGA forms the inner core, while the hydrophilic PEG arranges outside creating a stabilizing shell [15-17].

We have recently reported in vitro applications of lipophilic silver $(\mathrm{Ag})$-loaded PNPs derived from the PLGA- $b$-PEG-COOH block copolymer against glioblastoma cell lines [18]. We used the chlorotoxin (Cltx) as the targeting agent to show their in vitro targeting ability in the U87MG glioblastoma cell line. Cltx is a 36-amino acid-long peptide that specifically binds to MMP-2, a protease involved in remodeling the cell microenvironment, particularly the basement membrane $[18,19]$. Indeed, most research today is focused on achieving active targeting and therapeutic advantage of NPs by chemical modifications. To the best of our knowledge, no studies on the TDD of a combination of drugs and metallic NPs to treat in vivo malignant glioma have been reported.

In this study, we report the synthesis of PNPs containing the drug alisertib (Ali), a selective aurora $\mathrm{A}$ kinase (AAK) inhibitor and AgNPs, developed as a TDD system against GBM. An in vitro study on glioma cell lines and in vivo biodistribution and preliminary efficiency evaluations regarding tumor reduction are also described. To the best to our knowledge, this is the first study in which PNPs are radiolabelled with ${ }^{99 \mathrm{~m}} \mathrm{Tc}$ and imaged in vivo.

\section{Materials \& methods}

\section{Synthesis of Ag@PNPs}

The Ag@PNP nanosystem has been characterized previously [17]. Dynamic light scattering (DLS) showed a hydrodynamic diameter of $112.6 \pm 2.9 \mathrm{~nm}$ with a narrow size distribution (polydispersity index $[\mathrm{PDI}]=0.190 \pm 0.011)$ and $\mathrm{a} \zeta$-potential of $-35.3 \mathrm{mV}$. The concentration of Ag was measured by means of atomic absorption spectroscopy (AAS) and it was found to be $22799 \mathrm{ppm}$, corresponding to a $211.3-\mathrm{mM}$ solution.

\section{Synthesis of Ag@PNPs-Cltx}

For the Ag@PNPs-Cltx, DLS analysis showed a hydrodynamic diameter of $117.4 \pm 14.4 \mathrm{~nm}$ with a narrow size distribution $(\mathrm{PDI}=0.22)$ similar to the results obtained before Cltx conjugation. The $\zeta$-potential was $-16.2 \mathrm{mV}$ and the concentration of Ag was measured by AAS and was found to be $1402 \mathrm{ppm}$, corresponding to a $13.0-\mathrm{mM}$ solution.

\section{Synthesis of Ag/Ali@PNPs}

To synthesise Ag/Ali@PNPs, 50 mg of PLGA- $b$-PEG$\mathrm{COOH}$ (7 kDa PLGA/3 kDa PEG, $0.005 \mathrm{mmol}$ ) and $9 \mathrm{mg}$ of Ali $(0.017 \mathrm{mmol})$ were dissolved into a $1-\mathrm{ml}$ dispersion of AgNPs in dimethylsulfoxide (DMSO). The organic phase was mixed with $50 \mathrm{ml}$ of ultrapure water under vigorous stirring, maintaining a water:organic ratio of 10:1 with constant removal of the resulting solution. The mixture was kept under magnetic stirring for $30 \mathrm{~min}$ and then purified and concentrated using centrifugal filter devices (Amicon Ultra, Ultrace ${ }^{\circledR}$ membrane with 100,000 NMWL; Millipore, MA, USA) until the final volume of $5 \mathrm{ml}$. This dispersion was then filtered on a syringe filter Sterivex ${ }^{\mathrm{TM}}$ - GP polyether sulfone membrane with a $0.22-\mu \mathrm{m}$ pore size (Millipore) and stored at $4^{\circ} \mathrm{C}$. DLS analysis showed a hydrodynamic diameter of $190.6 \pm 0.8 \mathrm{~nm}$ and a PDI of $0.09 \pm 0.03$ with a $\xi$-potential of $-47.8 \pm 13.4 \mathrm{mV}$. $\mathrm{Ag}$ and Ali concentrations were determined using AAS and high-performance liquid chromatography (HPLC) analysis, respectively, and they were found to be $2280 \mathrm{ppm}$ of Ag, corresponding to 21 and $404 \mu \mathrm{M}$ of Ali.

\section{Synthesis of Ag/Ali@PNPs-Cltx}

$N$-hydroxysulfosuccinimide $(1.3 \mathrm{mg}, 11.0 \mu \mathrm{mol})$ and a solution of 1-ethyl-3-(3-dimethylaminopropyl) 
carbodiimide $0.28 \mathrm{M}(7.1 \mathrm{ml})$ were added to a suspension of Ag/Ali@PNPs (5 ml) in phosphate-buffered saline $(20 \mathrm{ml}, 0.01 \mathrm{M})$ under magnetic stirring. The mixture was left to react at room temperature for $30 \mathrm{~min}$ and then Cltx $(0.150 \mu \mathrm{g}, 0.038 \mu \mathrm{mol})$ dissolved in $1 \mathrm{ml}$ of water was added and the reaction mixture was allowed to react for an additional $8 \mathrm{~h}$. The mixture was then washed with phosphate-buffered saline solution three times and concentrated into centrifugal filter devices (Amicon Ultra, Ultracel membrane with 100.000 NMWL), to a final volume of $5 \mathrm{ml}$. Finally, Ag/Ali@PNPs-Cltx were filtered on a syringe filter Sterivex ${ }^{\mathrm{TM}}$-GP polyether sulfone membrane with a $0.22-\mu \mathrm{m}$ pore size and stored at $4^{\circ} \mathrm{C}$. DLS analysis showed a hydrodynamic diameter of $199.1 \pm 0.6 \mathrm{~nm}$ with a PDI of $0.21 \pm 0.02$ and a $\zeta$-potential of $-15.4 \pm 4.5 \mathrm{mV}$. The Ali concentration was determined using HPLC and was found to be $41.8 \mu \mathrm{M}$. An elemental analysis by atomic AAS gave an average $\mathrm{Ag}$ concentration of $2.17 \mathrm{mM}$.

\section{Trypan blue assay}

Cytotoxicity of Ali alone or PNPs either loaded with Ali (Ali@PNPs-Cltx) or with Ali and AgNPs (Ag/Ali@PNPs) were evaluated on U87MG using a trypan blue exclusion dye test. Cells were incubated for 48 and $72 \mathrm{~h}$ at concentrations of Ali ranging from 0.001 to $10 \mu \mathrm{M}$ (Supplementary Material, see online at www. futuremedicine.com/doi/suppl/10.2217/nnm.14.1). Data were analyzed as the percentage of viable cells against the control. Results obtained by the trypan blue assay were analyzed and expressed as the percentage of viable cells against the control (mean \pm standard error of the mean). Statistical analysis was performed applying the one-way ANOVA test and Dunnett's multiple comparison test. For each experimental point, six replicates and three independent experiments were performed. Linear regression analysis was performed by using STATGRAPHICS ${ }^{\circledR}$ Centurion XVI.

\section{Radiolabeling of the ${ }^{99 \mathrm{~m} T \mathrm{Tc}-\mathrm{NPs}}$}

Radiolabeling of PNPs was performed using the direct method according to a slightly modified previously described protocol [20,21]. Briefly, $40 \mu \mathrm{l}$ of an acidic, aqueous solution containing $\mathrm{SnCl}_{2}(10 \mathrm{mg}$ dissolved in $500 \mu \mathrm{l}$ of $\mathrm{HCl} 37 \%$, diluted to $10 \mathrm{ml}, 1 \mathrm{mg} / \mathrm{ml}$ ) was added to $100 \mu \mathrm{l}$ of pertechnetate eluate. The $\mathrm{pH}$ was adjusted to the range of 7 , with an aqueous solution of $\mathrm{NaHCO}_{3} 0.5 \mathrm{M}$. Finally, aliquots containing $2 \mu \mathrm{g}$ of NPs were added and the mixture was shaken horizontally at room temperature for $30 \mathrm{~min}$. Radioanalysis was performed using acetone and a mixture of pyridine:acetic acid:water (3:5:1.5) as mobile phases and instant thin layer chromatography medium-silica gel (ITLC-SG) sheets as the stationary phase.
Stability tests

Stability of the radiolabeled PNPs was assessed towards transchelation, using diethylenetriaminepentaacetic acid (DTPA) and histidine, two widely used chelators for ${ }^{99 \mathrm{~m}} \mathrm{Tc}$, and in plasma to assess their behavior in a biological medium. Thus, $50 \mu \mathrm{l}$ of each of the radiolabeled preparations was challenged against $450 \mu \mathrm{l}$ of histidine and DTPA solutions $(0.01 \mathrm{M})$ as well as against plasma. Each sample mixture was incubated in a water bath at $37^{\circ} \mathrm{C}$ for 1,3 and $6 \mathrm{~h}$, and was analyzed by ITLC-SG using acetone and saline as mobile phases for the DTPA/histidine challenge study, and acetone and a mixture of pyridine:acetic acid:water (3:5:1.5) as mobile phases for the plasma stability study.

Imaging studies in animal models

Radiolabeled PNPs (100 $\mu \mathrm{l}, 100-300 \mu \mathrm{Ci})$ were evaluated scintigraphically after bolus intravenous injection via the tail vein in healthy Swiss mice and severe combined immunodeficiency mice bearing U87MG tumors. All animal experiments were performed in compliance with the European legislation for animal welfare. Animals were anesthetized immediately after injection by the intraperitoneal injection of a proper anesthetizing solution $-0.5 \mathrm{ml}$ of $\mathrm{f}$ tamine hydrochloride $(100 \mathrm{mg} / \mathrm{ml}), 0.25 \mathrm{ml}$ of xyla $\bar{z}_{\alpha_{1}}$ e $(20 \mathrm{mg} / \mathrm{ml})$ and $4.25 \mathrm{ml}$ of $\mathrm{NaCl} 0.9 \%$ (dose: $0.1 \mathrm{ml} / 10 \mathrm{~g}$ of animal weight administered intraperitoneally). The animals were placed on the camera approximately 5 min after tracer injection and dynamic images of the anesthetized mice were obtained between 10 and at least $60 \mathrm{~min}$ post-injection using a high-resolution $\gamma$-camera system, which has been described elsewhere [22,23].

\section{Tumor decrease studies}

In the control group, no treatment was applied. In the Ag@PNPs-Cltx group, $100 \mu \mathrm{l}$ of a 5.97-mM AgNPs solution was injected. In the Ali@PNPs-Cltx group, $100 \mu$ of a 0.11 -mM Ali solution was injected and, in the Ag/Ali@PNPs-Cltx group, $100 \mu$ l of a solution containing 0.11-mM Ali (5.93 mM in Ag) was injected. All samples were injected at day 24 and were not radiolabeled in order to avoid further dilution and maximize the quantity of NPs and drug injected. The tumor size was calculated every day according to the formula $0.5 \times$ length $\times$ width ${ }^{2}$ and mouse weight was also measured until day 55 . Since five animals were initially used in each group, the mean value and the standard deviation were calculated for each group. Both values were extracted until the number of surviving animals decreased to three. The experiment ended at day 55 because animals in the control group, as well as other groups, started to die, and the tumor size was in some cases too large, meaning that animals had to be sacrificed. 


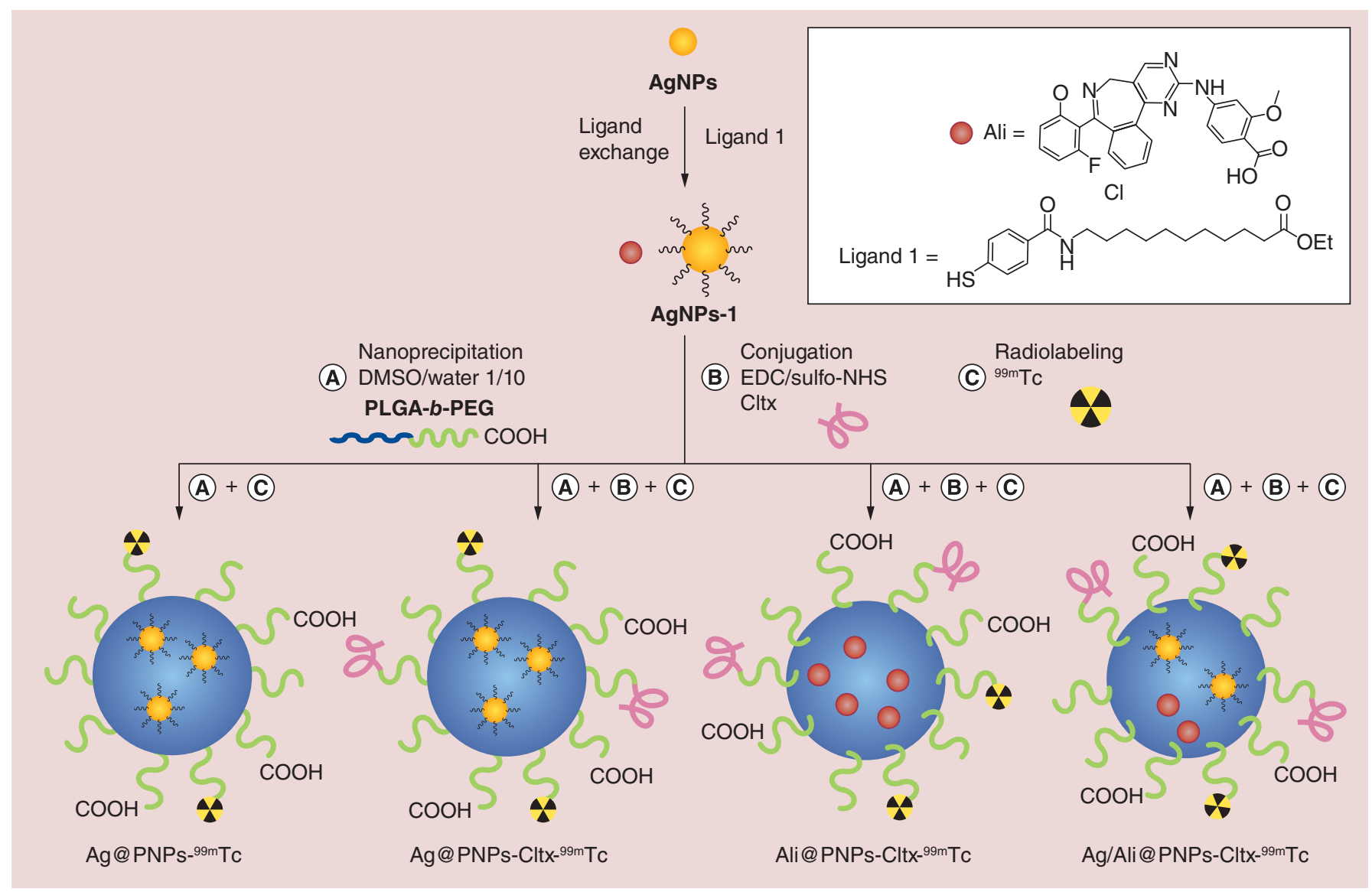

Figure 1. Synthesis of silver@polymeric nanoparticles-99mTc, silver@polymeric nanoparticles-chlorotoxin-99mTc, alisertib@polymeric nanoparticles-chlorotoxin-99mTc and silver/alisertib@polymeric nanoparticles-chlorotoxin-99mTc.

AgNP: Silver nanoparticle; Ali: Alisertib; Cltx: Chlorotoxin; DMSO: Dimethylsulfoxide; EDC: Ethyl(dimethylaminopropyl) carbodiimide; NHS: N-hydroxysuccinimide; PLGA-b-PEG: Poly(lactic-co-glycolic acid)-block-PEG-carboxylic acid; PNP: Polymeric nanoparticle.

\section{Results}

Chemistry \& nanotechnologies

Polyvinylpyrrolidone (PVP)-capped AgNPs were synthesized as reported in the experimental section. AgNPs were coated on their surface with ethyl 11-mercaptoundecanoate 1 , obtained as previously reported $[24,25]$ in order to make them lipophilic and stable in organic solvents and, thus, allowing their entrapment into the PNPs. Ligand 1 was designed with: a terminal thiol group that strongly binds to Ag; a connecting aliphatic chain that ensures stability in the system; and a terminal ester group in order to increase solubility in common organic solvents. After incubation of PVP-capped AgNPs with ligand 1, lipophilic AgNPs-1 were washed by centrifugation and redispersed in DMSO. The efficacy of this coating was previously proved with ${ }^{1} \mathrm{H}-\mathrm{NMR}$ and DLS analyses [18].

Next, AgNPs-1 were entrapped into PNPs using the nanoprecipitation technique [26]. The amphiphilic PLGA-b-PEG-COOH copolymer was selected to create biocompatible, biodegradable and water-soluble micelles able to circulate for long periods of time in the bloodstream. Therefore, the organic solution of AgNPs-1 and the copolymer was added dropwise to a larger amount of ultrapure water under vigorous stirring. The resulting Ag@PNPs were characterized as reported previously [18]. It is worth noting that, in this system, the AgNPs are preserved from dissolution by double-layer protection. The organic thiol, due to its high affinity for the particle surface and low exchange rate, stabilizes the surface, while the PLGA- $b$-PEG nanocarrier erects a defensive shell against potentially oxidizing agents (Figure 1) [27].

Exploiting the nanoprecipitation technique, Ali was entrapped into the same polymeric system. The DLS analysis of the obtained Ali@PNPs showed a hydrodynamic radius of $80.5 \pm 0.9 \mathrm{~nm}$ with a PDI of $0.120 \pm 0.004$ and a $\zeta$-potential of $-51.6 \mathrm{mV}$. Finally, the simultaneous entrapment into the same nanocarriers of both AgNPs-1 and Ali was investigated; we first dissolved Ali in dimethylsulfoxide containing AgNPs- 1 and copolymer, and then we used the nanoprecipitation techniques to create micelles. After purification, the Ag/Ali@PNPs were fully characterized; DLS analysis of this system showed a hydrodynamic diameter of $190.6 \pm 0.8 \mathrm{~nm}$ with a narrow size distribution $(\mathrm{PDI}=0.09 \pm 0.03)$ and $\mathrm{a} \zeta$-potential of $-47.8 \mathrm{mV}$. 
Once fabricated, all three nanosystems were conjugated with Cltx. Docking was achieved through amide bond formation between the carboxylic acids at the particle's surface and the free amine group of the peptide using the classical 1-ethyl-3-(3-dimethylaminopropyl) carbodiimide chemistry. The Ag@PNPs-Cltx, Ali@ PNPs-Cltx and Ag/Ali@PNPs-Cltx obtained were fully characterized using DLS, transmission electron microscopy and AAS (Figure 2).

Ag@PNPs-Cltx have already been reported and characterized by us [18]. DLS analysis of Ali@PNPs-Cltx confirms that the particle's dimensions are maintained with a hydrodynamic radius of $98.2 \pm 3.8 \mathrm{~nm}$ and a narrow size distribution $(\mathrm{PDI}=0.160 \pm 0.009)$. The $\zeta$-potential was found to be $-23.2 \mathrm{mV}$, and the Ali concentration was determined using HPLC analysis and was found to be 120.8 $\mu \mathrm{M}$. Regarding Ag/Ali@PNPs-Cltx, DLS analysis revealed a hydrodynamic diameter of $199.1 \pm 0.6 \mathrm{~nm}$ and a PDI of $0.210 \pm 0.018$. The $\zeta$-potential $(-15.4 \mathrm{mV})$ became less negative after Cltx conjugation. The Ali concentration was determined using HPLC analysis and was found to be $41.8 \mu \mathrm{M}$, while the Ag concentration was measured using AAS analysis and was found to be $234 \mathrm{ppm}$, corresponding to a $2.17-\mathrm{mM}$ solution.

\section{In vitro biological studies}

The effect of Ag@PNPs-Cltx on the U87MG human glioblastoma cell line and Balb/3T3 immortalized fibroblasts has already been reported, and cell-specific recognition of U87MG compared with Balb/3T3 cell lines, via Cltx, was observed. The uptake of Ag was also quantified and a cytotoxic effect corresponding to an $\mathrm{IC}_{50}$ of $45 \mu \mathrm{M}$ was found after $72 \mathrm{~h}$ of exposure [18]. In the present study, a comparison of these results with Ali alone and Ali@PNPs-Cltx, and the evaluation of the synergistic effect between AgNPs and Ali both loaded in micelles (Ag/Ali@PNPs-Cltx) was carried out.

The range of concentrations tested, related to the amount of Ali for all the compounds, was 0.001-10 $\mu \mathrm{M}$, corresponding to concentrations of $0.00005-0.5 \mu \mathrm{M}$ of $\mathrm{Ag}$ in Ag/Ali@PNPs-Cltx. Increasing concentrations and exposure times induced a statistically significant decrease in cell viability compared with the untreated cells (control: $100 \%$ cell viability) for all the compounds tested (Figure 3). DMSO was used to dissolve Ali and it was tested, as a solvent control, at a concentration of $0.2 \% \mathrm{v} / \mathrm{v}$. DMSO did not show any statistically significant toxicity when administered as the negative control. In fact, the cell viability after 48 and $72 \mathrm{~h}$ of exposure was 98 and 99\%, respectively. At each examined time point, Ali@PNPs-Cltx were more toxic than Ali alone; comparing the $\mathrm{IC}_{50}$ of Ali@PNPs-Cltx $(0.02 \mu \mathrm{M})$ and Ag/Ali@PNPs-Cltx $(0.01 \mu \mathrm{M}$ of Ali and $0.0005 \mu \mathrm{M}$ of $\mathrm{Ag}$ ), the latter was more toxic, but only after $72 \mathrm{~h}$ of incubation (Figure 3B). Furthermore, Ali@PNPs-Cltx showed a nonlinear dose-effect relationship after both 48 and $72 \mathrm{~h}$ of exposure with almost complete cell death at 5 and $10 \mu \mathrm{M}$; by contrast, the toxicity of Ag/Ali@PNPs-Cltx remained stable at approximately 45 and $30 \%$ cell viability at doses from 0.1 to $10 \mu \mathrm{M}$, after 48 and $72 \mathrm{~h}$ of exposure, respectively (Figure 3).

\section{Radiolabeling}

In vivo biodistribution was assessed using NPs radiolabeled with ${ }^{99 \mathrm{~m}} \mathrm{Tc}$ and high-resolution scintigraphic imaging. All of the products of this study were directly radiolabeled [21] with high efficiency $>95 \%$, and radiolabeled products demonstrated good stability properties. Transchelation against DTPA and histidine was evaluated by ascending ITLC-SG (Supplementary Material)

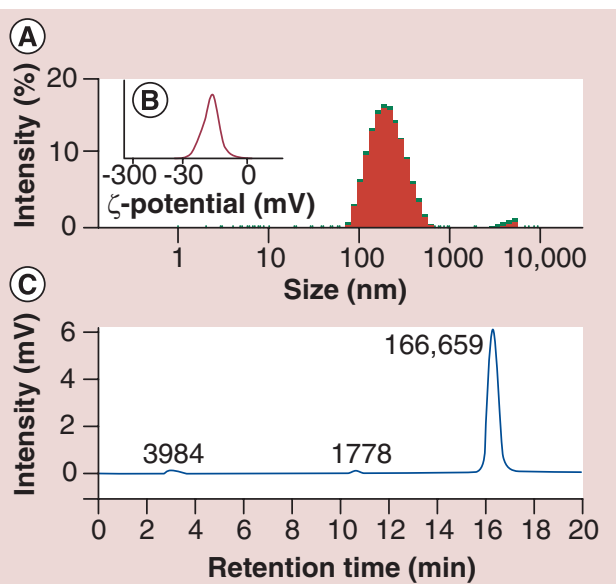

(D)

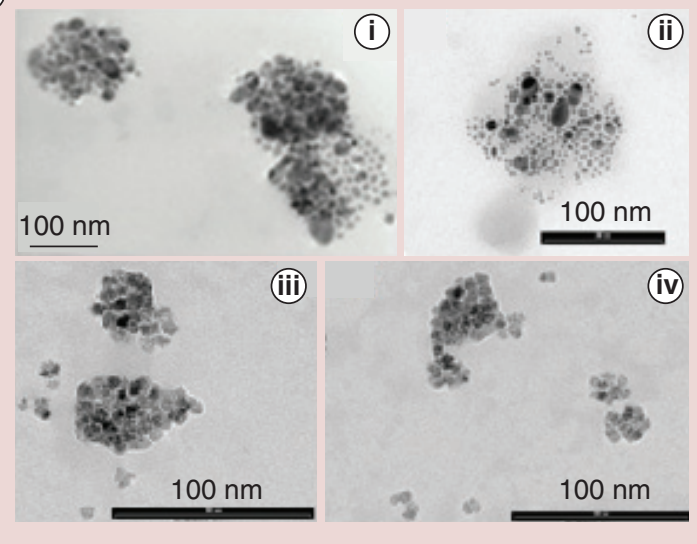

Figure 2. Analysis of silver/alisertib@polymeric nanoparticles-chlorotoxin. (A) Dynamic light scattering; (B) $\zeta$-potential; (C) high-performance liquid chromatography; and transmission electron microscopy images of (Di) silver (Ag)/alisertib@polymeric nanoparticles (PNPs)-chlorotoxin, (Dii) Ag/alisertib@PNPs, (Diii) Ag@PNPs-chlorotoxin and (Div) Ag@PNPs. 

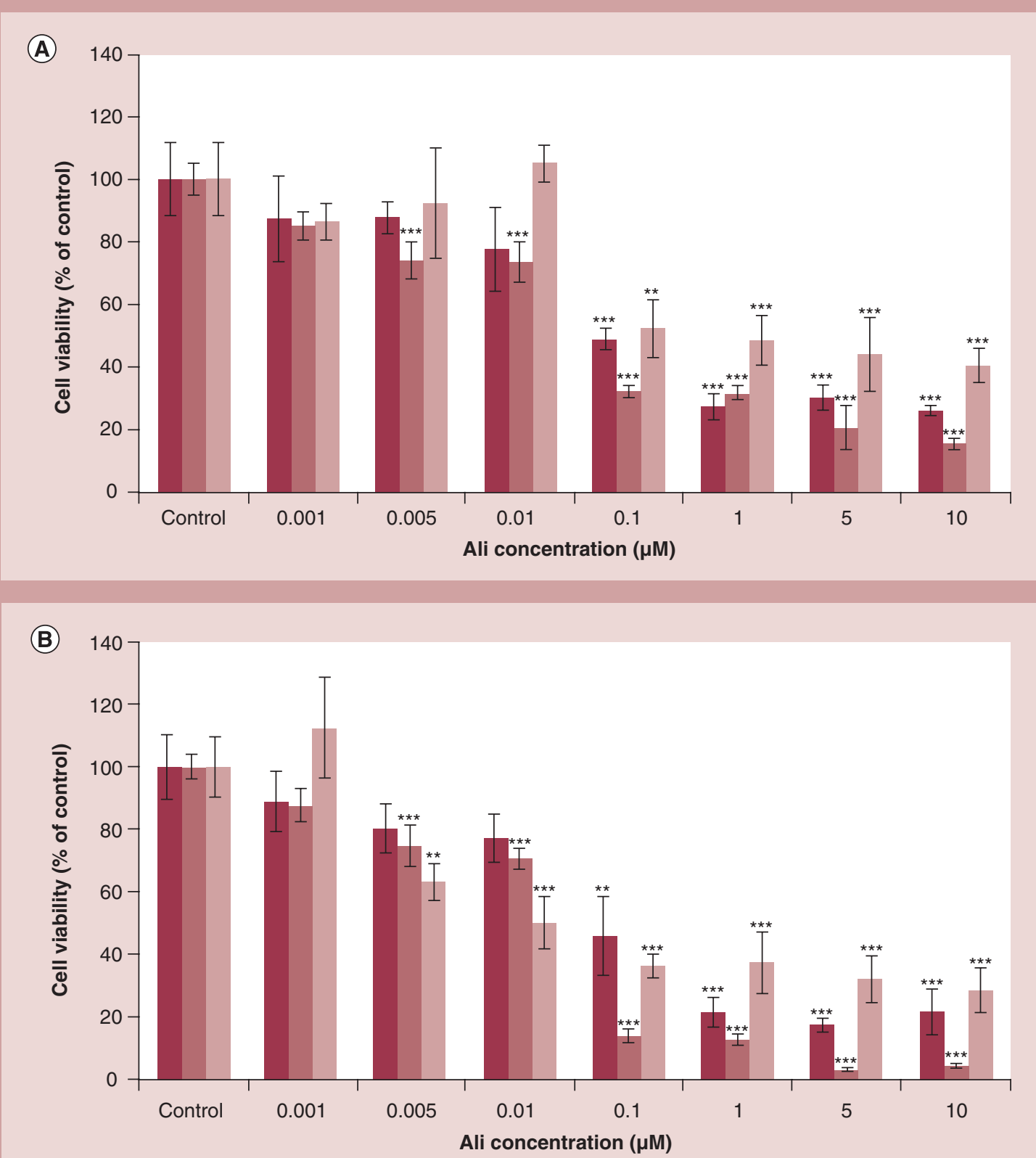

Figure 3. U87MG cells exposed to different polymeric nanoparticle formulations. U87MG cells exposed for (A) 48 and (B) 72 h to Ali, Ali@PNPs-Cltx and Ag/Ali@PNPs-Cltx at concentrations ranging from 0.001 to $10 \mu \mathrm{M}$ of Ali, corresponding to concentrations ranging from 0.00005 to $0.5 \mu \mathrm{M}$ of Ag contained in Ag/Ali@PNPs-Cltx. The IC $\mathrm{C}_{50}$ were $0.10,0.03$ and $0.10 \mu \mathrm{M}$ for Ali, Ali@PNPs-Cltx and Ag/Ali@PNPs-Cltx, respectively, at $48 \mathrm{~h}$ and $0.10,0.02$ and $0.01 \mu \mathrm{M}$, respectively, at $72 \mathrm{~h}$. Results are expressed as cell viability as a percentage of the control $(100 \%$ cell viability). A nonlinear dose- and time-dependent effect was observed for all the compounds tested. The in vitro data were analyzed using a $95 \% \mathrm{Cl}$ applied to a nonliner regression model.

$* * p<0.01 ; * * * p<0.001$.

Ag: Silver; Ali: Alisertib; Cltx: Chlorotoxin; PNP: Polymeric nanoparticle.

analysis using acetone and saline as mobile phases. In DTPA stability studies, after $1 \mathrm{~h}$ of incubation, all three NPs displayed moderate stability $68 \pm$ 4\% for Ag/Ali@

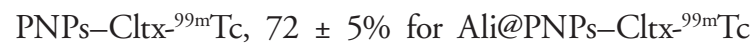
and $71 \pm 2 \%$ for Ag@PNPs-Cltx- ${ }^{99 \mathrm{~m}} \mathrm{Tc}$ ). It is worth noting that no reoxidation to pertechnetate occurred, but 
rather the instability was attributed to the formation of the stable ${ }^{99 \mathrm{~m}} \mathrm{Tc}-\mathrm{DTPA}$ complex. At $6 \mathrm{~h}$ post incubation, some intact radiolabeled NPs remained present (30 $\pm 0.8 \%$ for Ag/Ali@PNPs-Cltx- $-9 \mathrm{~m}$ Tc, $15 \pm 1.3 \%$ for Ali@PNPs-Cltx- ${ }^{99 \mathrm{~m}} \mathrm{Tc}$ and $20 \pm 0.9 \%$ for Ag@PNPsCltx- $\left.{ }^{99 \mathrm{~m}} \mathrm{Tc}\right)$. However, at this time point, the presence of significant amounts of $\mathrm{TcO}_{4}^{-}$was observed, at least for Ali@PNPs-Cltx- ${ }^{-9} \mathrm{~m} T \mathrm{Tc}(15 \pm 4 \%)$ and Ag@PNPs-Cltx${ }^{99 \mathrm{~m}} \mathrm{Tc}(41.5 \pm 5.5 \%)$. The three NPs remained almost intact $(>94 \%)$ after $1 \mathrm{~h}$ post-incubation, with no significant ${ }^{99 \mathrm{~m}} \mathrm{Tc}$ dissociation in favor of donor groups that are always present in plasma proteins. Nevertheless, further incubation resulted in the formation of larger radiocomplexes, as shown by the pyridine:acetic acid:water mixture quality control. At $6 \mathrm{~h}$ post incubation, only a fraction of radiolabeled NPs remained intact (31 $\pm 2.1 \%$ for Ag/Ali@ PNPs-Cltx- ${ }^{-99 \mathrm{~m}} \mathrm{Tc}, 17 \pm 1.9 \%$ for Ali@PNPs-Cltx- ${ }^{99 \mathrm{~m}} \mathrm{Tc}$ and $24 \pm 3.2 \%$ for Ag@PNPs-Cltx- ${ }^{99 \mathrm{~m}} \mathrm{Tc}$ ).

\section{In vivo biodistribution \& therapeutic effect}

In vivo studies in tumor-bearing mice have shown noticeable concentrations of the NPs in their tumors. Out of all tested products, the highest concentration was observed for the full system Ag/Ali@PNP-Cltx-

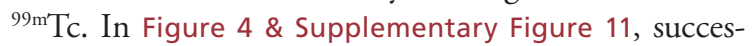
sive 2-min frames of a tumor-bearing mouse injected with Ag@PNPs- ${ }^{99 \mathrm{~m}} \mathrm{Tc}$ and Ag/Ali@PNP-Cltx- ${ }^{-99 \mathrm{~m} T \mathrm{c}}$ are shown. A significantly higher concentration of $\mathrm{Ag} /$ Ali@PNP-Cltx- ${ }^{-9} \mathrm{~m} T \mathrm{c}$ on the left shoulder, where the tumor is, compared with $\mathrm{Ag} @ \mathrm{PNPs}-{ }^{99 \mathrm{~m}} \mathrm{Tc}$ (without the targeting agent Cltx) can be observed.

Quantitative analysis (Supplementary Figures 11 \& 12) of the 2-min frames shows a concentration of $0.6 \%$ for Ag@PNPs- ${ }^{99 \mathrm{~m}} \mathrm{Tc}$ and 5\% for Ag/Ali@PNP_Cltx- ${ }^{99 \mathrm{~m}} \mathrm{Tc}$ in the tumor. This concentration is considered significantly different between the two formulations, as well as between Ag/Ali@PNP-Cltx- ${ }^{99 \mathrm{~m}} \mathrm{Tc}$ and normal tissue $(<2 \%)$. In addition, the concentration in the liver drops from 80\% for Ag@PNPs- ${ }^{99 \mathrm{~m}} \mathrm{Tc}$ to 60\% for Ag/Ali@ PNP-Cltx- ${ }^{99 \mathrm{~m}} \mathrm{Tc}$, and a noticeable and steady increase in the concentration in the bladder (from 3 to $7 \%$ ) for $\mathrm{Ag} /$
Ali@PNP-Cltx-99m Tc compared with Ag@PNPs- ${ }^{99 \mathrm{~m}} \mathrm{Tc}$ is observed.

For the initial assessment of a decrease in tumor size, 20 mice were divided into four groups, each one consisting of five animals - control, Ag@PNPs-Cltx, Ali@ PNPs-Cltx and Ag/Ali@PNPs-Cltx. The comparative measurements of the average tumor size and mouse weight for the four groups are given in Figure 5. These results are comparable and match those obtained in the in vitro test (Table 1).

\section{Discussion}

In this work we propose a new method for the synthesis of PNPs loaded with Ali and AgNPs to be used as an anticancer therapy.

All of the nanosystems reported have been obtained with an average diameter of less than $200 \mathrm{~nm}$, which is wildly recognized as a good range for drug delivery applications and medical purposes [28]. Ag/Ali@PNPs have a larger diameter that is probably caused by the simultaneous incorporation of two different agents into the PNPs, which remains a challenging task. The nanosystems have highly negative $\zeta$-potentials due to the presence of a large number of carboxylic acid groups on the micelle's outer shell. Negatively charged NPs have shown prolonged blood circulation and are generally less toxic compared with positively charged NPs [29,30]. After the conjugation reactions, the $\zeta$-potential was less negative, probably due to the loss of free carboxylic acids groups, which are now involved in the peptide conjugation.

Ali has been chosen as pharmacologic model for drug loading because of its effect as a selective AAK inhibitor and because its application against solid tumors (epithelial ovarian, fallopian tube and primary peritoneal carcinoma) is well known [31-33]. In this work, we also studied the synergic effect of Ali and AgNPs. AgNPs have already been proposed as anticancer agents because of their intrinsic toxic properties [34,35], and the AgNPs' cytotoxic effect against cancer cells has already been observed in vitro on U87MG cells, human leukemic K562 cells [36] and in vivo [37].
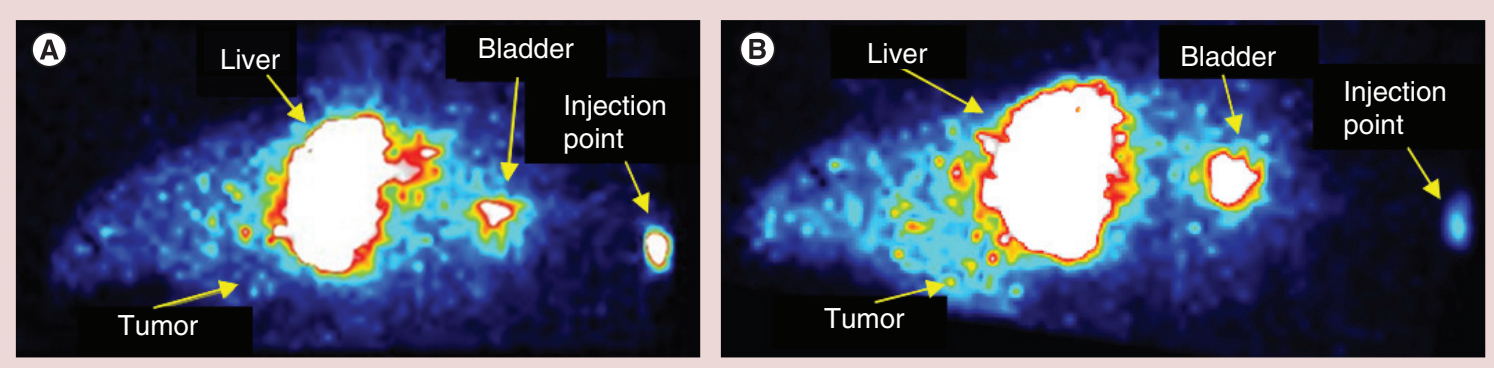

Figure 4. Comparative image of a tumor-bearing mouse injected with different polymeric nanoparticle formulations. (A) Silver@polymeric nanoparticles-99mTc and (B) silver/alisertib@polymeric nanoparticleschlorotoxin-99mTc post-injection. 


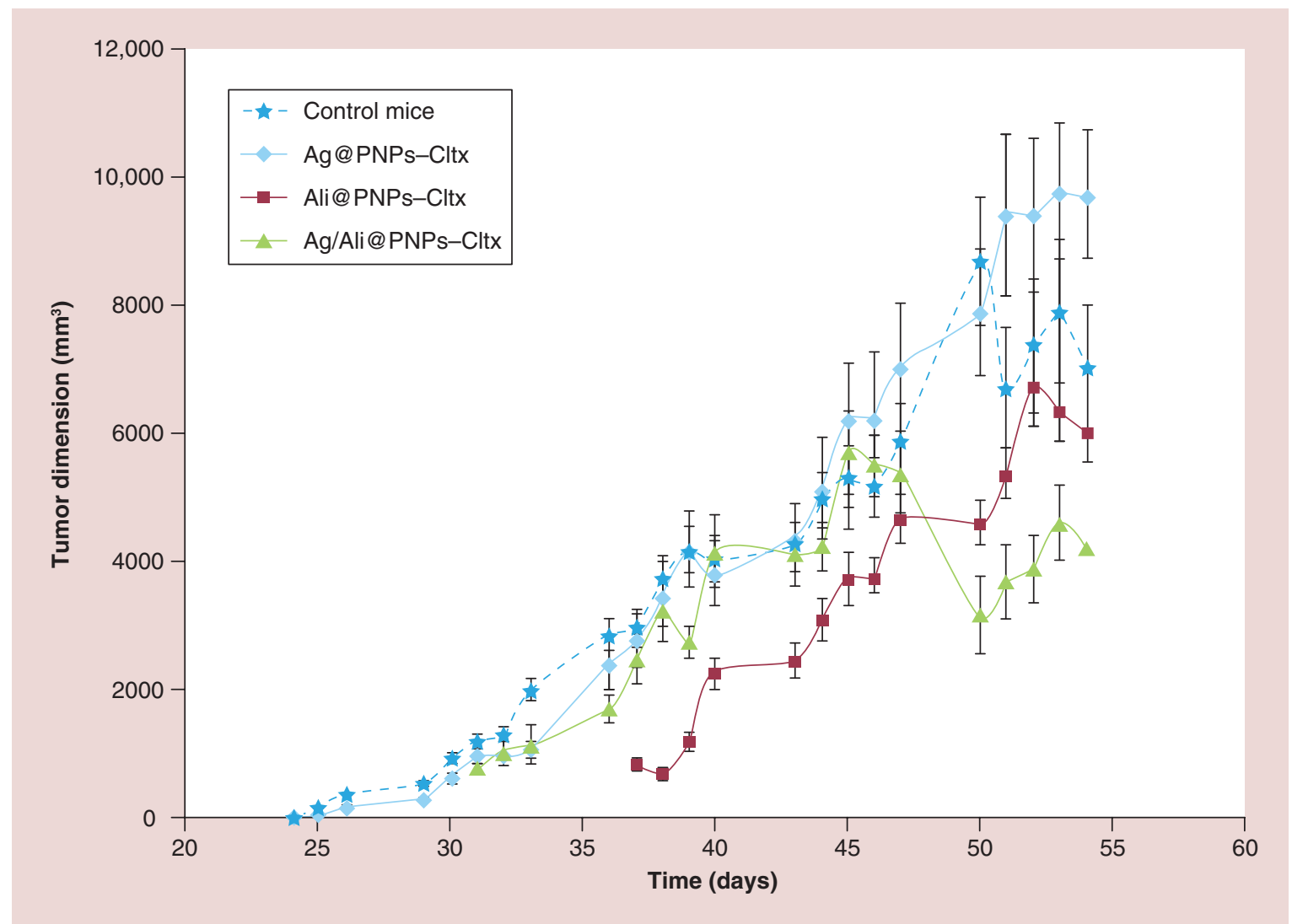

Figure 5. Tumor dimensions for the four tested mice groups. Control, Ag@PNPs-Cltx, Ali@PNPs-Cltx and Ag/Ali@PNPs-Cltx are shown. A decrease in tumor size is observed for Ag/Ali@PNPs-Cltx. Error bars represent the standard deviation.

Ag: Silver; Ali: Alisertib; Cltx: Chlorotoxin; PNP: Polymeric nanoparticle.

Regarding the stability of the radiolabeled NPs, DTPA and histidine challenges are the most efficient methods to determine stability. DTPA and histidine are considered two of the most efficient chelators for ${ }^{99 \mathrm{~m}} \mathrm{Tc}$, because they have the ability to complex with the radiometal in various oxidation states $[38,39]$. The moderate stability that all radiolabeled NPs present in DTPA after $1 \mathrm{~h}$ of incubation is expected considering the strong affinity that ${ }^{99 \mathrm{~m}} \mathrm{Tc}$ has for DTPA. Nevertheless, such conditions are never present in vivo, and, therefore, histidine challenge, which showed very good stability properties even after $6 \mathrm{~h}$ of incubation, provides more reliable evidence that these ${ }^{99 \mathrm{~m}} \mathrm{Tc}-\mathrm{NPs}$ will have good in vivo properties, since histidine is a molecule widely present in living organisms as a protein building material. Additionally, plasma stability studies are generally used as a means to indicate in vivo stability, since interaction with plasma proteins may alter the initial structure and/or characteristics of the radiolabeled NP, something that can ultimately result in the loss of its designed biological function. Plasma studies showed good stability properties of the radiolabeled NPs. The formation of larger radiocomplexes in plasma after $6 \mathrm{~h}$ may be attributed to the plethora of biomolecules existing in a biological environment that contain atoms and/or groups able to chelate and stabilize the ${ }^{99 \mathrm{~m}} \mathrm{Tc}$ core, or even compete for

\section{Table 1. Comparison between in vitro and in vivo results.}

\begin{tabular}{|lll|}
\hline Compounds tested & $\begin{array}{l}\text { In vitro in U87MG cells } \\
\left(I_{\mathbf{5 0}} ; \boldsymbol{\mu M}\right)\end{array}$ & $\begin{array}{l}\text { In vivo in glioblastoma-bearing mice (average } \\
\text { size change } \pm \text { standard deviation; \%) }\end{array}$ \\
\hline Ag@PNPs-Cltx & 45 & $+22 \pm 8.1$ \\
\hline Ali@PNPs-Cltx & 0.02 & $-22 \pm 6.1$ \\
\hline Ag/Ali@PNPs-Cltx & 0.01 & $-34 \pm 12$ \\
\hline Ali alone & 0.1 & Not determined \\
\hline $\begin{array}{l}\text { In vitro results are expressed as the IC } \mathrm{C}_{50} \text { obtained in U87MG cells after } 72 \mathrm{~h} \text { of incubation and in vivo in glioblastoma-bearing mice as the } \\
\text { observed average tumor size reduction after day 45. } \\
\text { Ag: Silver; Ali: Alisertib; Cltx: Chlorotoxin; PNP: Polymeric nanoparticle. }\end{array}$ \\
\hline
\end{tabular}


the limited NP surface, altering the NP's initial structure [40] and causing 'flocculation' of the NP.

The results of this study have revealed that, even after $6 \mathrm{~h}$ of incubation, the radiometal stays complexed on the NPs as no reoxidation to pertechnetate took place in the in vitro conditions that were chosen specifically to resemble those of the human body.

All imaging studies in vivo indicated, as expected, that NPs were present in the liver, as well as a slow - but continuous - increase in the concentration in bladder. The concentration in the tumor of the nontargeted $\mathrm{Ag} @ \mathrm{PNPs}-{ }^{99 \mathrm{~m}} \mathrm{Tc}$ was approximately $0.6 \%$ at $60 \mathrm{~min}$ post-injection, and this is almost certainly due to the enhanced permeability and retention effect. Taking into account that this value is almost double the concentration in normal tissue $(\sim 0.3 \%)$ we can say that this is quite encouraging for future studies.

For Ag/Ali@PNP-Cltx- ${ }^{-99 \mathrm{~m}} \mathrm{Tc}$, the concentration in liver decreased from 80 to $60 \%$ and, therefore, the effect of the targeting peptide is quite clear. This result, combined with the increased concentration in the bladder (from 3 to 7\%), provides strong evidence that $\mathrm{Ag} /$ Ali@PNP-Cltx- ${ }^{99 \mathrm{~m}} \mathrm{Tc}$ has favorable kinetic in vivo properties when compared with $\mathrm{Ag} @ \mathrm{PNPs}-{ }^{99 \mathrm{~m}} \mathrm{Tc}$, thus providing better scintigraphic images and as well as probably being more effective as a therapeutic agent. The potential of Ag/Ali@PNP-Cltx as a therapeutic delivery vehicle to cancer cells is also suggested by the significantly higher uptake of Ag/Ali@PNP_Cltx- ${ }^{99 \mathrm{~m}} \mathrm{Tc}$ compared with other ${ }^{99 \mathrm{~m}} \mathrm{Tc}-\mathrm{PNPs}$ in tumor tissue and the comparatively higher concentration of the nanoformulation in the tumor compared with normal tissue (Supplementary Figures 11 \& 12).

The results for tumor size show that treating the mice with Ag@PNPs-Cltx did not have a significant effect on tumor size. On the other hand, Ali@PNPs-Cltx resulted in a slowdown in tumor growth compared with the control and Ag@PNPs-Cltx groups. It should be noted that it was only possible to measure tumor size from day 36 and on the day of Ali@PNPs-Cltx injection. When Ag/Ali@PNPs-Cltx was injected, the development of the tumor was altered compared with the three other groups, and a decrease in tumor size was noticeable from day 48 . The weight of the mice was constant for all groups, except for the Ag/Ali@PNPs-Cltx group, where weight loss was observed after day 48, which correlates with the observed tumor decrease.

Data in Table 1 support the argument that a synergistic effect with Ag/Ali@PNPs-Cltx takes place. The in vitro results at $72 \mathrm{~h}$ and the in vivo effect after day 48 showed that the decrease in tumor size was greater than with the other nanosystems [41].

It is still uncertain and currently under debate whether the toxicity of AgNPs is a particle-specific effect [42] or whether it is caused by the dissolution of $\mathrm{Ag}^{+}$ions $[43,44]$. In our system, the synergistic effect may be based on the coexistence of the drug with $\mathrm{Ag}^{+}$ ions formed from slow AgNPs dissolution, once they are released from the protective polymeric shell.

Since the synergic effect of drugs and NPs could have some advantages in the drug delivery and therapeutics field, it will be important in the near future to better investigate this both in vitro and in vivo, maybe also increasing the $\mathrm{AgNP}$ concentration and analyzing different effects induced by these nanomaterials, as well as assessing alternative administration protocols.

\section{Conclusion}

In conclusion, we have reported the synthesis of some novel theranostic agents containing the small-molecule Ali and AgNPs. ${ }^{99 \mathrm{~m}} \mathrm{Tc}-$ radiolabeling of the nanocarrier allowed us to see the in vivo biodistribution in U87MGtumor bearing mice, the $\mathrm{IC}_{50}$ at $72 \mathrm{~h}$ and the tumor reduction in vivo that was obtained with Ag/Ali@ PNPs-Cltx, which couples the two agents together and promotes a synergistic effect. These results convey the potential of this novel theranostic agent for TDD.

\section{Future perspective}

The potential of targeted NPs for cancer treatment is immense, and a variety of systems have been developed and studied. However, systems bearing multiple therapeutic agents with a synergistic effect on tumor reduction are relatively novel. Thus, more studies are needed to explain the synergistic effect of the two cytotoxic agents. The understanding of this mechanism will allow the development of novel systems bearing multiple components with synergistic effect for the treatment of different types of cancer.

\section{Financial \& competing interests disclosure}

This work was supported by the University of Bologna. This work was also supported by the Work program Action 15024: Nanobiotechnologies of DG Joint Research Centre. The authors have no other relevant affiliations or financial involvement with any organization or entity with a financial interest in or financial conflict with the subject matter or materials discussed in the manuscript apart from those disclosed.

No writing assistance was utilized in the production of this manuscript.

\section{Ethical conduct of research}

The authors state that they have obtained appropriate institutional review board approval or have followed the principles outlined in the Declaration of Helsinki for all human or animal experimental investigations. In addition, for investigations involving human subjects, informed consent has been obtained from the participants involved. 


\section{Background}

- Glioblastoma multiforme (GBM) is a malignant primary brain tumor with a bleak prognosis. The survival time without treatment is 3 months.

- Nanomedicine and targeted drug delivery are a powerful approach for cancer treatment.

Materials \& methods

- Synthesis of nanocarriers containing silver nanoparticles with alisertib have been developed.

- Resulting polymeric nanoparticles have been conjugated with the peptide chlorotoxin and radiolabeled with ${ }^{99 \mathrm{~m} T c}$.

- In vitro toxicity against GBM cell lines showed a synergistic effect between silver and alisertib.

- In vivo radiolabeling showed a GBM-targeted biodistribution of the nanoparticles and a therapeutic effect.

Conclusion \& future perspective

- A targeted strategy using a polymeric nanoparticles for drug delivery against GBM has been developed.

- This approach may be applied for future nanomedicine applications.

\section{References}

Papers of special note have been highlighted as:

- of interest; $\bullet$ of considerable interest

1 Furnari FB, Fenton T, Bachoo RM et al. Malignant astrocyticglioma: genetics, biology, and paths to treatment. Genes Dev. 21, 2683-2710 (2007).

-• Important review regarding glioma.

2 Siegel R, Naishadham D, Jemal A. Cancer statistic. $C A$ Cancer. J. Clin. 62, 10-29 (2012).

3 Weingart J, Grossman SA, Carson KA et al. Phase I trial of polifeprosan 20 with carmustine implant plus continuous infusion of intravenous $\mathrm{O}_{6}$-benzylguanine in adults with recurrent malignant glioma: new approaches to brain tumor therapy CNS Consortium Trial. J. Clin. Oncol. 25(4), 399-404 (2007).

4 Stupp R, Mason WP, van den Bent MJ et al. Radiotherapy plus concomitant and adjuvant temozolomide for glioblastoma. N. Engl. J. Med. 352, 987-996 (2005).

5 de Groot JF, Fuller G, Kumar AJ, Piao Y, Eterovic KJY, Conrad CA. Tumor invasion after treatment of glioblastoma with bevacizumab: radiographic and pathologic correlation in humans and mice. Neuro-Oncology 12(3), 233-242 (2010).

6 Addeo R, Caraglia M, De Santi S et al. A new schedule of fotemustine in temozolomide-pretreated patients with relapsing glioblastoma. J. Neurooncol. 102, 417-424 (2011).

7 Kohler BA, Ward E, McCarthy BK et al. Annual report to the nation on the status of cancer, 1975-2007, featuring tumors of the brain and other nervous system. J. Natl Cancer Inst. 103, 714-736 (2011).

8 Wagner V, Dullaart A, Bock AK, Zweck A. The emerging nanomedicine landscape. Nat. Biotech. 24, 1211-1217 (2006).

9 Kawasaki ES, Player A. Nanotechnology, nanomedicine, and the development of new, effective therapies for cancer. Nanomedicine 1, 101-109 (2005).

10 Koo OM, Rubinstein I, Onyuksel H. Role of nanotechnology in targeted drug delivery and imaging: a concise review. Nanomedicine 1, 193-212 (2005).

11 Freitas RA. What is nanomedicine? Nanomedicine 1, 2 (2005).
12 Kreuter J. Nanoparticles as drug delivery systems. In: Encycolpedia of Nanoscience and Nanotechnology (Volume 7). Nalwa HS (Ed.). American Scientific Publishers, CA, USA, 161-180 (2004).

-. Discusses nanoparticle formation, characteristics and advantages.

13 Lamprecht A, Ubrich N, Yamamoto $\mathrm{H}$ et al. Biodegradable nanoparticles for targeted drug delivery in treatment of inflammatory bowel disease. J. Pharmacol. Exp. Ther. 299, 775-781 (2001).

14 Gregoriadis G. Drug entrapment in liposomes. FEBS Lett. 36, 292-296 (1973).

15 Hrkach J, Von Hoff D, Ali MM et al. Preclinical development and clinical translation of a PSMA-targeted docetaxel nanoparticle with a differentiated pharmacological profile. Sci. Transl. Med. 4, 128 (2012).

16 Gu F, Zhang L, Teply BA et al. Precise engineering of targeted nanoparticles by using self-assembled biointegrated block copolymers. Proc. Natl Acad. Sci. USA 105, 2586-2591 (2008).

17 Locatelli E, Comes Franchini M. Biodegradable PLGA- $b$ PEG polymeric nanoparticles: synthesis, properties and nanomedical applications as drug delivery system. J. Nanop. Res. 14, 1316 (2012).

- Examines poly(lactic-co-glycolic acid)-block-PEGcarboxylic acid copolymer.

18 Locatelli E, Broggi F, Ponti J, Marmorato P, Lena S, Comes Franchini M. Lipophilic silver nanoparticles and their polymeric entrapment into targeted-PEG-based micelles for the treatment of glioblastoma. Adv. Healthcare Mat. 1, 342-347 (2012).

- Demonstrates the synthesis, characterization and anti-tumor effect of silver nanoparticles.

19 Deshane J, Garner CG, Sontheimer H. Chlorotoxin inhibits glioma cell invasion via matrix metalloproteinase-2. J. Biol. Chem. 278, 4135-4144 (2003).

- Explains the biological role of chlorotoxin.

20 Veiseh O, Sun C, Gunn J et al. Optical and MRI multifunctional nanoprobe for targeting gliomas. Nano Lett. 5, 1003-1008 (2005). 
21 Psimadas D, Baldi G, Ravagli C et al. Preliminary evaluation of a ${ }^{99 \mathrm{~m}} \mathrm{Tc}$ labeled hybrid nanoparticle bearing a cobalt ferrite core. J. Biomed. Nanotechnol. 8(4), 575-585 (2012).

22 Psimadas D, Bouziotis P, Georgoulias P, Valotassiou V, Tsotakos T, Loudos G. Radiolabeling approaches of

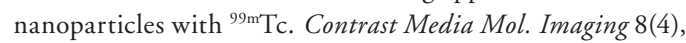
333-339 (2013).

- Discusses radiolabeling with ${ }^{99 \mathrm{~m}} \mathrm{Tc}$.

23 Loudos G, Majewski S, Wojcik R et al. Performance evaluation of a dedicated camera suitable for dynamic radiopharmaceuticals evaluation in small animals. IEEE Trans. Nucl. Sci. 54(3), 454-460 (2007).

24 Gentili D, Ori G, Comes Franchini M. Double phase transfer of gold nanorods for surface functionalization and entrapment into PEG-based nanocarriers. Chem. Comm. 39, 5874 (2009).

25 Ponti J, Lemor R, Fournelle M, Broggi F, Locatelli E, Comes Franchini M. Polymeric entrapped thiol-coated gold nanorods: cytotoxicity and suitability as molecular optoacoustic contrast agent. J. Mat. Chem. 20, 10908-10914 (2010).

26 Bonini BF, Camaggi CM, Comes Franchini M et al. Design and synthesis of novel 3, 4-disubstituted pyrazoles for nanomedicine applications against malignant gliomas. Eng. J. Med. Chem. 45, 2024-2033 (2010).

27 Liu J, Sonshine D, Shervani S, Hurt RH. Controlled release of biologically active silver from nanosilver surfaces. ACS Nano 4 , 6903-6913 (2010)

28 Albanese A, Tang PS, Chan WC. The effect of nanoparticle size, shape, and surface chemistry on biological systems. Ann. Rev. Biomed. Eng. 14, 1-16 (2012).

29 Nemmar A, Hoylaerts MF, Hoet PH et al. Ultrafine particles affect experimental thrombosis in an In vivo hamster model. Am. J. Respir. Crit. Care Med. 166, 998-1004 (2002).

30 Kedmi R, Ben-Arie N, Peer D. The systemic toxicity of positively charged lipid-nanoparticles and the role of Toll-like receptor 4 in immune activation. Biomaterials 31, 6867-6875 (2010).

31 Matulonis UA, Sharma S, Ghamande S et al. Phase II study of MLN8237 (alisertib), an investigational aurora A kinase inhibitor, in patients with platinum-resistant or -refractory epithelial ovarian, fallopian tube, or primary peritoneal carcinoma. Gynecol. Oncol. 127(1), 63 (2012).

- Investigates alisertib's mode of action and efficiency.

32 Cervantes A, Elez E, Roda D et al. Phase I pharmacokinetic/ pharmacodynamic study of MLN8237, an investigational, oral, selective aurora A kinase inhibitor, in patients with advanced solid tumors. Clin. Cancer Res. 18(17), 4764-4774 (2012).

33 Dees EC, Cohen RB, von Mehren M et al. Phase I study of aurora a kinase inhibitor MLN8237 in advanced solid tumors: safety, pharmacokinetics, pharmacodynamics, and bioavailability of two oral formulations. Clin. Cancer Res. 18(17), 4775-4784 (2012).

34 Jeyaraj M, Sathishkumar G, Sivanandhan G et al. Biogenic silver nanoparticles for cancer treatment: an experimental report. Colloids Surf. B Biointerfaces 106, 86-92 (2013).

35 Guo D, Zhu L, Huang Z et al. Anti-leukemia activity of PVP-coated silver nanoparticles via generation of reactive oxygen species and release of silver ions. Biomaterials 34(32), 7884-7894 (2013).

36 Wu Q, Cao H, Luan Q et al. Biomolecule-assisted synthesis of water-soluble silver nanoparticles and their biomedical applications. Inorg. Chem. 47, 5882-5888 (2008).

37 Asharani PV, Wu YL, Gong Z, Valiyaveettil S. Toxicity of silver nanoparticles in zebrafish models. Nanotechnology 19 , 255102-255110 (2008).

38 Eckelman WC, Richards P, Meinken G. The chemical state of ${ }^{99 \mathrm{~m}} \mathrm{Tc}$ in biomedical products. II. The chelation of reduced technetium with DTPA. J. Nucl. Med. 13, 577-581 (1972).

39 Rattat D, Terwinghe C, Verbruggen A. Comparison of 'classic' ${ }^{99 \mathrm{~m}} \mathrm{Tc}-\mathrm{DTPA},{ }^{99 \mathrm{~m}} \mathrm{Tc}$ (CO) 3-DTPA and ${ }^{99 \mathrm{~m}} \mathrm{Tc}$ (CO) 2 (NO)-DTPA. Tetrahedron 61(40), 9563-9566 (2005).

40 Psimadas D, Oliveira H, Thevenot J et al. Polymeric micelles and vesicles: biological behavior evaluation using radiolabeling techniques. Pharm. Dev. Technol. 19(2), 189-193 (2014).

41 Caraglia M, Longo L, Salzano G et al. Stealth liposomes encapsulating zoledronic acid: a new opportunity to treat neuropathic pain. Mol. Pharmaceutics 10, 1111-1118 (2013).

42 Pratsinis A, Hervella P, Leroux JC, Pratsinis SE, Sotiriou GA. Toxicity of silver nanoparticles in macrophages. Small 9(15), 2576-2584 (2013).

43 Kittler S, Greulich C, Diendorf J, Koeller M, Epple M. Toxicity of silver nanoparticles increases during storage because of slow dissolution under release of silver ions. Chem. Mater. 22, 4548-4554 (2010).

44 Xiu ZM, Zhang QB, Puppala HL, Colvin VL, Alvarez PJJ. Negligible particle-specific antibacterial activity of silver nanoparticles. Nano Lett. 12, 4271-4275 (2012).

\section{Website}

101 ClinicalTrials.gov www.clinicaltrials.gov 